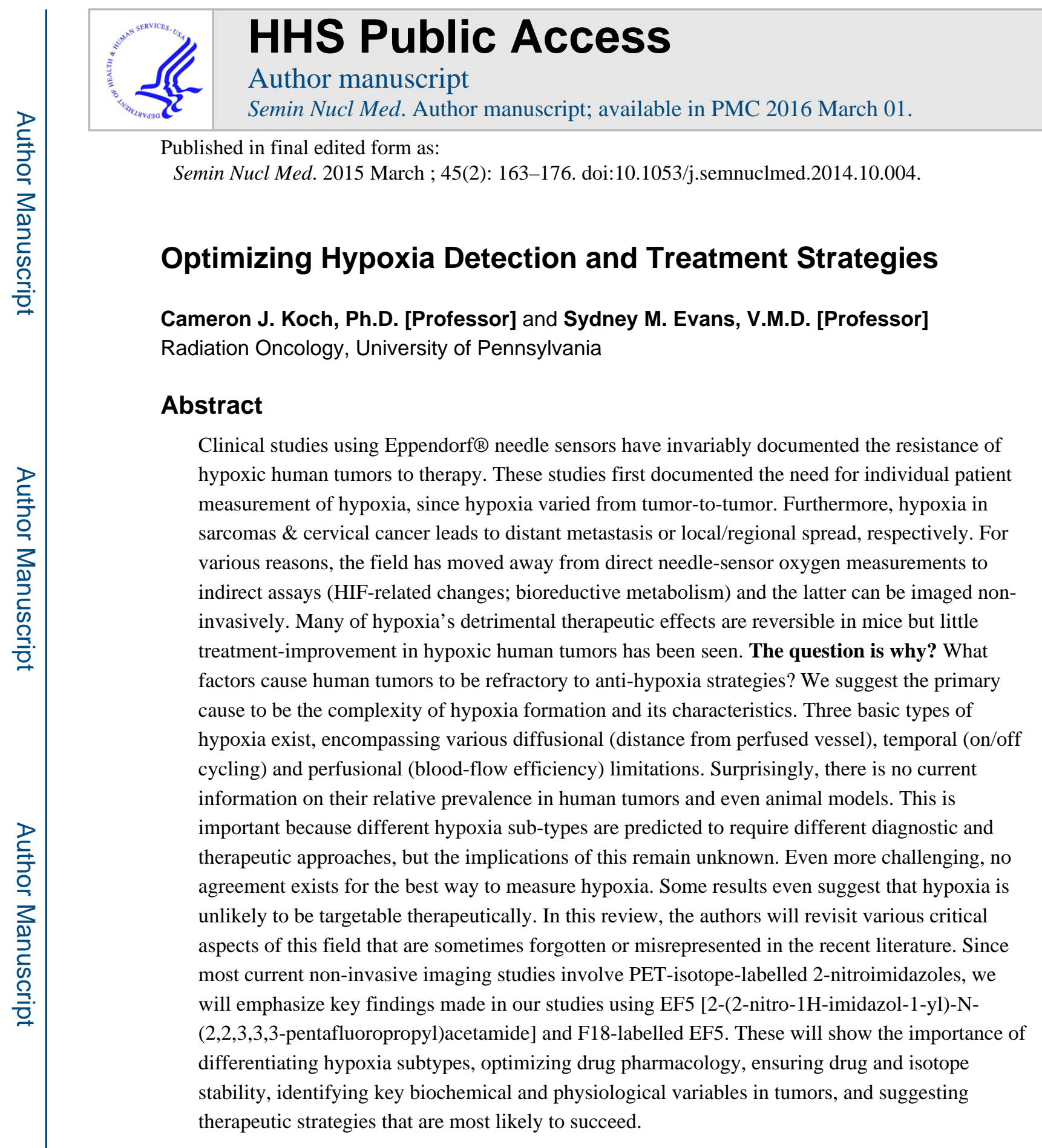

\title{
What is 'Hypoxia'?
}

In this review, we will document various complexities in our understanding of tumor hypoxia (oxygen levels less than normal tissue) and how it could be best measured to

(C) 2014 Elsevier Inc. All rights reserved.

Address for Proofs: Rm 8-130, SCTR Bldg. 421, 3400 Civic Ctr Blvd., Philadelphia, PA 19104-5156. Contact Info: kochc@mail.med.upenn.edu, phone 215 898-0073; fax 215 898- 5573

Publisher's Disclaimer: This is a PDF file of an unedited manuscript that has been accepted for publication. As a service to our customers we are providing this early version of the manuscript. The manuscript will undergo copyediting, typesetting, and review of the resulting proof before it is published in its final citable form. Please note that during the production process errors may be discovered which could affect the content, and all legal disclaimers that apply to the journal pertain. 
optimize therapy. In the past, hypoxia has often been described as if it were a binary phenomenon (oxygen 'present' versus 'absent') but in practice one must consider physiological factors that produce a complete range of tissue $\mathrm{pO}_{2}$ 's. In the body, arterial $\mathrm{pO}_{2}$ is typically stated to be in the range of $75 \mathrm{~mm}$ of $\mathrm{Hg}$ with venous blood averaging 40 $\mathrm{mm}$ of $\mathrm{Hg}$. We generated a logarithmically decreasing scale (Table 1) to provide a frame of reference for discussion of various 'degrees' of hypoxia (1). This table has some biological basis; HIF1-a is upregulated at $\mathrm{pO}_{2}$ 's below mild hypoxia (2); commonly studied cell lines are roughly half-maximally resistant to ionizing radiation at 'moderate' hypoxia (3); cells typically have metabolic problems when exposed to severe hypoxia and below, since 0.76 $\mathrm{mm}$ of $\mathrm{Hg}$ is roughly the $\mathrm{K}_{\mathrm{m}}$ for respiration (2). As will be seen in this review, identification of the degree of hypoxia required to produce the various types of therapy resistance in humans is not known with any degree of accuracy. Hopefully, this situation will improve with future research.

\section{Section 1: Basic aspects of tumor physiology cause three types of hypoxia}

\section{a. Diffusion-Limited Hypoxia (DLH) and Consequential Research}

In 1955, Thomlinsom and Gray made the pioneering suggestion that human epithelial tumors might be hypoxic and hence radioresistant (4). Their suggestion was based upon the appearance of tumor-derived tissue-sections (see Fig. 1a), with distance of blood vessels from central necrosis in tumor cords being consistent with the diffusion properties of oxygen (no hypoxia assays existed for human tumors at the time). A substantial preclinical research effort ensued in the 60's and 70's to develop oxygen-mimetic radiosensitizers and hypoxiaspecific cytotoxins $(5,6)$ that would target presumably radioresistant tumors. These compounds appeared very efficacious in murine models, but subsequent clinical trials showed little improvement in patient outcome $(7,8)$. Hypoxia research underwent a nadir in the 1980's with a major consideration being that hypoxic therapy resistance might be a problem limited to rodent tumors. This pessimistic view was fundamentally reversed by clinical studies using oxygen needle-sensors made by the Eppendorf company $(9$, and sarcomas $(\mathrm{N} \& \mathrm{H}) \mathrm{d}$ and neck cancer Studies in cervical and hea .(10 unequivocally showed that hypoxia represented a universal therapy resistance mechanism and in some the presence of hypoxia predicted distant metastasis or (cervical cancer \&sarcomas .g.e)types -tumor sensor studies directly showed that -the needle, Critically .

$(12,11,9)$ respectively, vasionregional in/local tumor hypoxia wasnot a common feature of specific cancer sub-types (e.g. H\&N cancer) but rather varied unpredictably both within individual tumors and on a tumor-to-tumor basis. This fundamental observation is crucial for therapeutic progress, since anti-hypoxia therapeutic trials would need 8-fold more patients to determine a significant impact on a mixed population of aerobic and hypoxic tumors (13). Additionally, individual tumor assessment of hypoxia is critical to target therapy to those patients that need it, while sparing morbidity to those that do not.

While the needle-sensor studies were very successful in predicting therapy resistance, it is important to recognize that the criteria used to define hypoxia differed between studies. Although these differences could have been caused by the technical challenges involved 
with the measurements, there was also the indication that 'hypoxia' was not the same in all tumors $(14,15)$.

\section{b. Cycling Hypoxia (CH)}

In the 1980's a second 'type' of hypoxia was suggested, confirming a prediction made by Dr. J. Martin Brown several years earlier (16). Termed acute or cycling hypoxia, it was demonstrated in animal tumor models by intravenous injection of two fluorescent DNAbinding dyes. Both dyes are rapidly removed from the circulating blood (minutes) since they bind strongly to DNA in cells of the vessel lumen and adjacent tissue. After tumor removal and sectioning, the dyes could be distinguished by their emission color. When the dyes were injected simultaneously, all observed vessels were labelled by both dyes but when the dyes were injected individually at times separated by about 20 minutes a small fraction of vessels were labeled by one but not both dyes $(17,18)$. These vessels were interpreted to have had a change of blood flow during the time between injections (see figure 1b). It is important to recognize that 'cycling-hypoxia' is somewhat of a misnomer since first, this assay is not an actual measure of hypoxia, rather it marks blood flow over two very short time periods, and secondly, that the dyes used (typically Hoechst 33342 and DiOC7) are known to be vasoactive. In other words, they can directly cause the effect that is being measured (9). $\mathrm{CH}$ has important therapeutic and diagnostic implications but the DNA-binding dyes cannot be used in humans - thus there is no current information on its presence in human tumors. In fact the fraction of vessels subject to cycling flow has not been well characterized even in rodent tumor models.

\section{c. Macroscopic Regional Hypoxia (MRH)}

We recently described a 3rd type of hypoxia using the EF5 hypoxia marker [2-(2-nitro-1Himidazol-1-yl)-N-(2,2,3,3,3-pentafluoropropyl)acetamide] (20). Although DLH and CH are expected to produce small and medium-sized isolated hypoxic zones (respectively), we noted orders of magnitude larger regions of hypoxia (EF5 binding) in human GBM tumors and sarcomas $(21,22)$. These regions included large numbers of apparently functional vessels (since the tissue was viable). MRH appears to be a 'super-sized' version of a phenomenon originally described by Dewhirst and colleagues in a rat window-chamber model (23). This type of tumor is confined to the vascular bed of subcutaneous tissue and can be up to a few $\mathrm{mm}$ in diameter but less than $0.1 \mathrm{~mm}$ thick. In this model, individual arterioles were shown to develop oxygen gradients along the path of flow, described as 'longitudinal arteriole gradients'. MRH was also found in the 9L gliosarcoma grown in isogenic rats $(20,24)$ and the group at Duke University has recently published an example in a murine xenograft of epithelial cancer, both by PET-autoradiography and immunohistochemistry (IHC) (25). MRH is most easily observed in large tumors due to their geometrical requirements for provision of blood flow throughout the entire tumor mass. Normal tissue is organized with pairs of vessels carrying countercurrent arterial and venous blood. In contrast for tumors, individual vessels and their branches are unpaired and can extend for many millimeters through the tumor mass. These result in extended longitudinal gradients (ELG) of nutrients, especially including oxygen, and even therapeutics along their length (Fig 1c). This has the important consequence that the blood flowing through hypoxic regions is deoxygenated and may actually be a sink for tissue oxygen - in turn, the locally- 
produced VEGF can permeabilize the vessels and this can, for the first time, provide a physiological explanation for the propensity of hypoxic tumors to metastasize. In much smaller 3-5 mm diameter murine tumors (particularly subcutaneous), MRH may manifest itself as central necrosis since the small-caliber vessels arising from the skin and subcutaneous tissues in mice are poorly adaptable for carrying blood over long distances (see Fig 1d). Demonstrating that there actually is blood flow within MRH is challenging because blood-flow markers (e.g. the DNA-binding dyes as described for $\mathrm{CH}$ ) are themselves consumed along the extended vessel-gradients - we found that fluorescent dextrans, which are not consumed by binding, were suitable for this task (20). Of course, an indirect proof is the viability of the cells in these regions - the complete absence of blood flow will always lead to cell death similar to that seen in stroke and vascular disease (26). In addition to possibly explaining metastasis, two very clinically relevant aspects of MRH are 1) it represents a fundamental difference between large versus small tumors and 2) when tumors are grown in orthotopic location, they have access to whole-organ blood supply that can support the larger caliber vessels and longer gradient lengths involved.

To summarize, tumor hypoxia is an important aspect of the tumor microenvironment. It arises when oxygen supply from the vasculature is less than oxygen consumption by the tumor tissue. This imbalance is very simple to describe but details of its control are complex and poorly understood, not only from one tumor to the next but also within each tumor. Nevertheless, clinical studies using needle-sensors (Eppendorf®) have directly demonstrated that hypoxia represents a universal therapy resistance mechanism. In some tumor-types (e.g. sarcomas \& cervical cancer) hypoxia predicts distant metastasis or local/regional spread, respectively. Critically, the needle-sensor studies showed that tumor hypoxia was not a universal feature of specific cancer types (e.g. all head and neck cancers) but varied on a tumor-to-tumor basis. Thus, the presence and extent of hypoxia needs assessment in each tumor to optimize/personalize therapy.

\section{Section 2: Hypoxia Detection Methods}

Since the 1990's, it has been recognized that expanded use of the needle-sensors was impractical despite their original importance (e.g. in some cases they are too dangerous to monitor primary tumors, only lymph nodes, and they usually can't reach deep-seated tumors). Thus, a number of new (but indirect) assays for hypoxia have been developed (molecular assays related to HIF upregulation (27), EPR methods $(28,29), \& 2-$ nitroimidazole binding - 30). Many are applicable in humans and some (EPR and 2nitroimidazole binding) allow non-invasive imaging (NII). With the exception of EPR methods, no existing method, including the needle sensors, can sample hypoxia as a function of time. Unfortunately, EPR methods do not presently have enough spatial resolution to detect $\mathrm{CH}$, at least for isolated vessels as described above, and cannot work in the vast majority of human cancers because the RF frequencies are too high to penetrate tissue very far. This review will therefore focus on 2-nitroimidazole binding.

The sensitizer trials of the 1970's and -80's (use of nitroimidazoles to make tumors more radiosensitive) had mostly negative clinical results except for the use of the 5-nitroimidazole nimorazole in Denmark, for H\&N cancer - (31). After hypoxia-dependent toxicity by the 
sensitizers was discovered (32, 33), Varghese and colleagues showed that bioreductive metabolism of these drugs led to covalent adducts between their metabolites and cellular (macro)molecules (34). This result was further developed by Chapman and colleagues who suggested the use of sensitizers as tissue-hypoxia imaging agents (35). His work and that of several others emphasized NII using SPECT (single photon emission computed tomography - 36) and PET technologies (37), while the labs of Raleigh, Hodgkiss, and Koch developed CCI-103F \& pimonidazole, NITP, and EF5 and EF3, respectively, as probes for the immunohistochemical (IHC) detection of hypoxia (38-41). EF5 and EF3 have also been made with one fluorine atom labeled for PET imaging - $(42,43)$. The first F-18-labelled drug to be clinically tested was FMISO and it remains the most extensively tested agent (for review see 37, 44). Due to some perceived problems with FMISO's stability in vivo (45) and failure to achieve image intensities in humans comparable to what had been achieved in animal models, or for example the very high intensity contrast seen with FDG, many additional nitroimidazoles have been synthesized for the purpose of NII and several have progressed from preclinical development to therapeutic trials. These include FETNIM, FETA, IAZGP, EF5, EF3, IAZA, FAZA and HX4 and others - for review of structures and properties see Horsman (46).

\section{Oxygen-dependence of 2-nitroimidazole binding}

Most of the original biochemical characterization of oxygen-dependent binding arose more than 20 years ago using C-14-labelled misonidazole and etanidazole (47-49). A qualitative summary is illustrated in Figure 2. As can be seen for the curves in the upper right of this figure, most data were generated in the 3 to $100 \mu \mathrm{M}$ range, due to limitations in counting afforded by the very low specific activity (SA) of C14 compared to PET isotopes. For 'wellbehaved' compounds such as etanidazole, binding is first-order in drug concentration, and obeys simple inhibition kinetics with respect to oxygen concentration $(50,51)$.

The form of the oxygen dependence of drug binding is:

$$
\text { BindingRate }=\mathrm{k}_{\mathrm{dr}} \times[\mathrm{drug}] \times\left[\mathrm{K}_{\mathrm{i}} /\left(\left[\mathrm{O}_{2}\right]+\mathrm{K}_{\mathrm{i}}\right)\right]
$$

where $\mathrm{k}_{\mathrm{dr}}$ is a cell and drug related factor related to nitroreductase levels and $\mathrm{K}_{\mathrm{i}}$ is the oxygen concentration for half-maximal binding rate. Due to the complexities of specifying the local oxygen concentration, this is typically listed with units of oxygen partial pressure $(\mathrm{mm}$ of $\mathrm{Hg}$ ) or gas phase concentration (\%, considering '100\%' as dry oxygen gas at one atmosphere pressure).

Despite the simplicity of this formula there are several complicating factors. First, this only applies to one-electron reductases. Any other form of metabolism, especially including 2electron reduction, can cause an additional non-oxygen dependent component of metabolism (52). Secondly, there are variations in maximum binding rate with different drugs $\left(\mathrm{k}_{\mathrm{dr}}\right)$. Thirdly, some compounds (e.g. misonidazole) are not 'well-behaved' in that they exhibit half-order dependence of binding on drug concentration, so binding rate decreases by the square root of ten per 10-fold decrease in drug concentration. However, this only occurs at extremely low oxygen levels. Since all drugs studied to date have binding rates that are first- 
order in drug concentration at intermediate levels of hypoxia, this can cause huge uncertainties in what might be happening at the drug concentrations commonly employed for PET in humans ( $\sim 0.1 \mathrm{nM}$ for high SA F-18). In fact, there are no data in the world literature that specify what the binding characteristics of any of these drugs are at the sub$\mathrm{nM}$ concentration range. Thus, extrapolation of kinetic data for even the 'well-behaved' compounds must be considered speculative (Fig 2; dashed lines). For this reason, Chapman has suggested the inclusion of a few micromolar unlabelled drug with NII (48). In a recent study by Busk et al, the authors have partially bridged this gap using F18-labelled FAZA at approximately $15 \mathrm{nM}$ (53) with a culture system employing glass dishes, shaken under controlled gas conditions (54). In their data (estimated with an arrow and open triangle in Fig 2) the intracellular bound FAZA was 40-fold higher than the extracellular concentration of free drug for hypoxic conditions, but equal to the extracellular concentration for aerobic conditions - after rinsing, both drug concentrations decreased but the ratio of remaining drug in hypoxic versus aerobic cells increased to about 200. This is very exciting data but unlike the in vitro situation, there is no excess extracellular pool of drug in vivo. Thus, one might expect severe diffusion limitations for this drug under conditions of severe hypoxia.

The elephant hiding in the room for use of these agents is variability in tissue levels of nitroreductases. The BindingRate equation is written as if $\mathrm{k}_{\mathrm{dr}}$ is a constant, but it could be mixed since there are several one-electron reductases present in mammalian cells (55). Few of the mammalian enzymes have been characterized as a function of drug concentration, and none at the low drug concentrations used for PET. However, Prosser and colleagues have investigated several bacterial nitroreductases and found $\mathrm{K}_{\mathrm{m}}$ ' $\mathrm{s}$ in the low $\mathrm{mM}$ to $\mu \mathrm{M}$ range (56) - see Table 2. Due to these relatively high values, it is probably safest to assume that all drugs have first-order concentration dependence of binding. The octanol/water partition coefficient of a particular 2-nitroimidazole may have the effect of emphasizing specific nitro-reductases, depending on their local environment and this may even affect the $\mathrm{K}_{\mathrm{i}}$, since oxygen is quite lipophilic - hence its concentration varies with environment by a factor of 10 or more. In the process of nitroreduction, only the first electron transfer is thought to play a role in the oxygen dependence of binding. That is because the resulting nitro-radical anion is not very reactive and hence has an opportunity to transfer the electron back to oxygen (the oxygen-dependent step). Once an additional electron is added (nitroso) the drug becomes a much stronger oxidizing agent and is likely committed to an overall 4-electron reduction (hydroxylamine), but the source of the additional 3 electrons and the process is unclear (57, 58). Once the hydroxylamine is formed, the reduced drug can react with either (soluble) non-protein sulfhydryls or high molecular weigh protein thiols (59). In IHC assays for drug adducts, the cells or tissue sections are fixed, so any low molecular weight products or adducts are lost, but their egress from cells and tissues has not been documented in vivo. For IHC, the source of signal arises from the protein adducts. Raleigh and Koch showed that the fraction of metabolized drug that was bound to macromolecules increased with lipophilicity (57). Thus, signal strength is lower for hydrophilic compounds but the impact of this result on PET imaging is not known. Similarly, hydrophilic compounds preferentially partition with the extracellular rather than intracellular space (60). This has the effect of decreasing the intracellular drug concentration so this again decreases the amount of bound drug. The impact of these two synergistic deficiencies of hydrophilic compounds is unknown in vivo. 


\section{Section 3: Expected Hypoxia and Nitroreductase Levels in Human Tumors}

Perhaps least discussed in the literature for imaging of hypoxia is the 'degree' of hypoxia found in human tumors. We developed EF5 as an IHC marker with the specific goal of trying to quantify both the distribution of hypoxia and its severity in multiple tissue types (26). The quantification process was to evaluate the total drug exposure (via HPLC measurements of plasma after drug injection and at surgery) and the maximum binding rate using small pieces of tumor tissue incubated in vitro under hypoxic conditions (cubereference binding) $(1,26)$. The only assumption was that variations in binding reflected the same oxygen dependence as was seen in vitro. Using this system, we found that severe hypoxia was rare in animal tumors (61). In humans, we found that almost all low grade tumors have no hypoxia at all (though many cancer types have not been studied with this technique) (62) - with the exception of $\mathrm{H} \& \mathrm{~N}$ cancer (where hypoxia can be found in all grades) only high grade tumors contain regions with significant degree's of hypoxia (see table 1 for definitions). Similarly, most normal tissue appears to be completely welloxygenated (exceptions are skin which has pockets of severe hypoxia near some sebaceous glands and hair follicles) and liver which contains mild hypoxia (1). We are fairly confident in these measurements due to EF5's lipophilic nature - it exists everywhere at basically the same concentration as in blood. The EF5 results demonstrate the presence of even less hypoxia than did the Eppendorf studies, but the trend was the same (i.e. that hypoxia was less severe in human than animal tumors). Even in tumors that have regions of severe hypoxia, most of the tumor volume sampled is characterized by physiological levels of oxygenation.

The two labs that have most thoroughly addressed the issue of variability in nitroreductase levels are the Koch/Evans labs at University of Pennsylvania and the Wilson/Patterson labs at University of Auckland. The latter have validated several enzymes for their interests in using bioreductives as therapeutics against tumors with hypoxia. The most widely distributed of these enzymes is cytochrome P450 reductase (POR) - see Table 2. Due to the flexibility in determining binding using EF5 (based on either radioactive drug uptake or IHC) the Auckland group has investigated the relationship between binding of EF5 and activation of SN30000 (a next generation analog of tirapazamine). Despite wide ranges of overall nitroreductase levels in their extensive panel of cells, EF5 was found to track SN30000 activation more closely than levels of POR alone (63). This will be discussed further in the clinical trials section, below. EF5's structure was based on etanidazole, since early studies with this compound (using C14-labelled drug uptake) showed the least variability in binding using a broad variety of cell lines $(47,49)$. However, no other structure-function relationships have been undertaken since the 1990's to determine if drugs with even more consistent binding are possible. Particularly as a result of the Auckland team's work, it is now clear that the metabolism of all similar drugs is dependent on nitroreductase levels - its just that most have not been tested in detail. We speculate that any drugs suggested to be have binding independent of nitroreductase levels are likely to suffer from either non-oxygen-dependent metabolism or an as yet unknown mechanism of activation and binding. 
Due to the relatively recent identification of mammalian nitroreductase enzymes, there are very few studies that have looked at the variability in this property of tumor tissue. Using EF5 at the concentrations needed for IHC analysis $(100 \mu \mathrm{M}$ and $67 \mu \mathrm{M}$ whole-body in animals and humans, respectively) we have accounted for this factor, as discussed above, by employing the 'cube-reference binding' assay (CRB) to determine the maximum binding rate for each tissue assessed $(1,26)$. The CRB studies produced a highly significant result there were roughly 20 -fold variations in tumor tissue nitroreductase levels in more than 150 tumors studied (it should be noted that the CRB assay is also impacted somewhat by cellularity - see below. If one considered only high-grade tumors that were most likely to contain hypoxia, as well as those tumors having relatively high cellularity, this variation was less than 10 . Nevertheless, the range of difficulty in identifying potentially therapy-resistant tumors might go from tumors with isolated pockets of DLH and low-nitroreductase levels to those with MRH and high levels of nitroreductase. We expect that knowledge of a tumor's nitroreductase levels should improve the interpretation of patient-to-patient variability in apparent image contrast but there is no data to support or refute this expectation. Similarly, we do not presently know whether variations in nitroreductase occur at the level of the specific tissue or the whole organism.

We have explored this question in a pilot study to see whether CRB in the skin overlying a tumor varied in a way similar to that of the tumor (1). This was found to be the case over the range of about 5 found in this limited study of 8 patients, but since the skin was taken from a different anatomical region in each patient, we do not know if skin binding is the same everywhere for an individual patient. If nitroreductase levels do vary consistently on a patient-to-patient basis, then it might be possible to assess a mildly hypoxic normal tissue to provide an appropriate control. In our hands skin is the only normal tissue consistently found to contain small regions of severe hypoxia and clearly skin can always be included in NII. However, little is known about this property of skin and the total amount of hypoxic tissue in skin is sufficiently small that dynamic scan analysis would be required. An alternative approach might be to assess binding in the white cell component of a blood sample, or to do a molecular analysis of nitroreducatse expression in a biopsy or blood sample, once this process is better understood. Additionally, the concerns over nitroreductase levels have only been demonstrated at the relatively high drug concentrations required for IHC. There is no current information on the oxygen and tissue dependence of binding at the very low drug concentrations used for imaging of high SA drugs such as FMISO.

\section{Section 4 - Early to Present Investigations of NII of Hypoxia}

Taking account of the sensitivity of 2-nitroimidazole binding to inhibition by relatively low levels of oxygen (Table 1) and lack of severe hypoxia in human tumors, the relatively lowcontrast images found in initial trials of F18-labelled FMISO are entirely appropriate. At the University of Washington, Rasey, Krohn and colleagues noted this in their studies and additionally pointed out the importance of the wide variation in cellularity for human tumors. Thus they have championed the requirement for detection of relatively small changes in SUV with voxels greater than 1.4 being 'positive' for hypoxia (64). This has been extended in studies by Thorwarth and colleagues, who have emphasized kinetic analysis of FMISO uptake - this accounts for the variable rates of blood-tissue equilibrium 
with this compound (65). Interestingly, in this study the worst-responders were identified as a group of patients with combined hypoxia and poor perfusion. It is tempting to speculate that the tumors of these patients might have MRH. FMISO has undergone extensive testing in a large number of trials that are summarized by relatively recent reviews $(44,46)$.

Of course, much of the above described information has only become available recently. Thus, many new imaging agents have been tested with the overall goal to provide greater sensitivity and specificity for tumor hypoxia than was provided by FMISO. Essentially, this requires improving the signal in hypoxic tumors, while decreasing the background in aerobic tissue.

The criteria involved with the development of these compounds have followed 3 distinct and relatively incompatible hypotheses;

1. Improve relative tumor uptake by using isotopes with half-lives comparable to or longer than the pharmacological half-life of the hypoxia-sensing drug. This generally requires iodinated compounds.

2. Improve relative tumor uptake by emphasizing rapid clearance of the parent compound from normal tissues. This requires hydrophilic compounds with an F-18 label

3. Emphasize uniform drug distribution and accept the resulting parent drug background, with image quality enhanced through more sophisticated imaging analysis (e.g. looking at the kinetics of uptake -(65). This requires lipophilic compounds.

There appear to be three main problems with the first hypothesis and so it will be discussed minimally. The first is that deiodination inevitably occurs. This is equivalent to a nonoxygen dependent metabolism, and results in the non-specific redistribution of the isotope. Secondly, the relevant iodine isotopes for PET are not pure positron emitters. This has the effect of degrading image quality and increasing patient dose. Thirdly, it is not practical to arbitrarily increase imaging times; this means, for longer-lived isotopes, a substantial increase in patient dose in order to observe the same counting rates after longer times. The most detailed study combining all aspects of this approach is for the use of IAZGP in the thorough study done by the Memorial Sloan-Kettering group. In this study, significant binding was not observed in a patient population where at least $50 \%$ of the tumors were expected to contain hypoxia (66).

Other factors being equal hypotheses 2) and 3) depend on the pharmacological properties of the drugs. One of the more distracting lessons imprinted on the research community from the sensitizer trials was that lipophilic drugs were more toxic than hydrophilic drugs (67). Since the normal tissue at risk was brain and peripheral nerves, it seemed reasonable that one would like to minimize blood-brain-barrier crossing, a well-known property of hydrophilic drugs. We have previously noted that this general principal is, in fact, not the whole story since desmethylmisonidazole was much more toxic than etanidazole, even though both were similarly hydrophilic (i.e. octanol:water partition coefficients $\sim 0.05)(30)$. Thus, molecular structure seems to be a factor of equal or even greater importance than 
lipophilicity. Nevertheless, reduction of potential toxicity of 2-nitroimidazole imaging agents through the development of hydrophilic drugs has been a common theme for many years (68). We suggest that this should be a non-issue for NII due to the low drug concentrations used (typically $0.1 \mathrm{nM}$ for high SA F18, versus $\mathrm{mM}$ concentrations used in sensitizer trials).

Another factor in favor of hydrophilic drugs (hypothesis 2) is the suggestion that hydrophilic drugs will have improved contrast between drug metabolism (uptake) and parent drug elimination through renal clearance of the parent compound. Hydrophilic drugs generally have much shorter biological half-lives than their lipophilic counterparts. However all imaging drugs under current study have clearance times in humans significantly longer than the half-life of the most chemically stable isotope (F-18). Since imaging must occur before even one biological half-life has passed, it is difficult to see how this factor could add more than a few percent to image contrast. Even though hydrophilic drugs clear more quickly than their lipophilic counterparts, they may also have reduced metabolism due to the effects described in the previous section, and additionally, they take much longer to achieve equilibrium between blood and tissue. This makes for very complex analysis of binding kintetics $(69,70)$. For delivery of drugs that are being consumed by tissue, hydrophilic drugs are at a significant disadvantage compared with drugs that are lipophilic (71). This effect was first noted in elegant experiments by Sutherland and colleagues who were investigating the ability of adriamycin to bind to and kill the inner cells of multicellular spheroids (72).

A critically-important demonstration of these principles was made by the Turku research group in a rat model. They were studying the biodistribution of FETNIM, a very hydrophilic analogue of misonidazole, but rather than simply assessing gamma counts in various tissues at various times, they extracted the tissue and analyzed the radioactive products by HPLC. They found that there was no detectable parent drug in liver and very little in tumor (73). They suggested that tumor uptake of this compound must be by a different mechanism than the normal bioreductive metabolism and binding but this mechanism remains unknown. We had made a somewhat similar observation (unpublished), comparing EF5 and FMISO distribution in rat liver - whereas parent EF5 was present in liver tissue at higher concentrations than blood, parent FMISO concentration was close to undetectable (except for the roughly $10 \%$ component associated with blood content). Using EF5 at concentrations appropriate for IHC (e.g. $100 \mu \mathrm{M}$ ) we showed many years ago that liver was not very hypoxic and bound little drug (particularly in animals larger than mice). Yet liver always seems to have high uptake in PET images. In the case of EF5 (both humans and rats) liver always has almost twice as much drug as does blood, but this is true immediately after injection and the overall decay has the same pharmacological lifetime as for blood (74). This cannot be the result of bioreductive metabolism - otherwise EF5 would be cleared by the liver in essentially first-pass metabolism, whereas we mainly see parent drug in this tissue. Thus, we think this reflects simple partitioning of drug into the lipophilic environment of liver tissue. Exclusion of hydrophilic compounds like FETNIM from the liver may involve a vessel-wall-based barrier somewhat akin to the blood-brain barrier, though this has not previously been discussed in the pharmacology literature. It would make sense for the body to direct, by passive means, lipophilic drugs to the liver and hydrophilic drugs to the kidney. 
Alternatively, there may be an active transport system that removes hydrophilic drugs from liver tissue. We originally thought that this problem with liver diffusion was structural, not pharmacological, since it was initially found only in analogues of misonidazole (FETNIM, FMISO). However, we have subsequently determined that EF1, which has a partition coefficient essentially identical to FMISO but with a structure analogous to etanidazole is also largely excluded from liver (Koch, Evans \& Kachur; unpublished data).

Hypothesis 3) suggests that lipophilic drugs are required in order to achieve rapid and uniform tissue access. The effect of this is to provide a constant level of parent drug, supplemented by a time dependent increase in the bioreductive signal. To illustrate this an orthotopic R3230 mammary tumor was grown in a syngeneic female Fischer rat until it reached a size of about $2 \mathrm{~g}$. Under isoflurane anesthesia, the rat's body temperature was maintained at $37^{\circ}$ and unlabelled + labeled EF5 were administered via an in-dwelling tail catheter. Three hrs later the tumor was removed, 3 minutes following an injection of Hoechst 33342. The tumor was immediately frozen, sectioned and assessed for labeled EF5 by autoradiography (AR). Based on in vitro data, one would expect the signal to consist of parent drug, metabolized drug that is not bound, and metabolized drug that is bound (Fig. 3, left panel). One can distinguish the bound drug by fixing the tissue, as would be done for immunohistochemistry (Fig. 3, right panel). This image was enhanced 4 fold to provide the same visual intensity. The image arising from the fixed tissue was then compared with IHC data (Fig. 4). Antibodies to bound EF5 give an image that is essentially the same as the fixed-tissue AR image. The only way to distinguish between the metabolites and parent drug lost in the rinsing step would be to concentrate the fixing solution (ethanol) and assess by HPLC.

There are a few literature examples of this type of experiment (generally using unlabelled pimonidazole as the IHC marker - 53) but to our knowledge, the comparison of non-fixed versus fixed AR sections has not been previously investigated. Nevertheless good correlations of the AR and IHC signal have sometimes been observed which indicates to us that free drug is generally at low levels in these studies. This would generally be the case in mice, due to the very short biological half-life of drugs in this species (compared with larger animals or humans).

An example using EF5 was provided in an illustrative study by the Duke group (25). They were comparing PET, AR and IHC in 3 different tumor models, two in mice and one in rats. They found a very good correspondence of AR and IHC for the two murine tumors, but a low-contrast image (like Fig. 3, left panel) for the rat image. This reflects the pharmacology of the drug in the two species: EF5 has a drug half-life of 50' in mice but 150' in rats. The images were derived at $3 \mathrm{hr}$ following injection for all three tumor types so most parent drug was gone in the mice, but still near half its original value for the rat. This paper directly tests the concept that one can get improved image contrast by decreasing the biological half-life of the imaging drug, not by using a lipophilic and hydrophilic drug but by using species with long and short drug half-lives. Interestingly, the PET images looked similar for the three tumors - this should be expected since in the rat, the drug concentration remains higher throughout the adduct formation time. In humans, the biological half-life of all imaging drugs is longer or much longer than the isotope half-life for F18. Thus, human images 
should be expected to consist of a mix of parent drug and metabolized drug, unless the parent drug has insufficient diffusional access to the tissue. We cannot expect the same levels of image contrast for images in humans as for animals, particularly mice. This suggests to us that the decreased ability of hydrophilic compounds to attain tissue equilibrium is most likely to result in decreased image contrast.

With respect to the three types of hypoxia described in the first section of this review there is unfortunately little information on either their prevalence or differential ability to be imaged.

Using IHC detection of EF5, MRH has clearly been observed in two of the most therapeutically resistant human tumors (GBM and high-grade sarcomas - 21, 22). These tumor types are prone to regional spread and metastasis, respectively. Thus, it is important to ensure that the 'geometrical' explanation we have provided (Fig 1c - based on the size of the hypoxic regions and inclusion of many vessels) is not complicated by other factors: for example, could MRH be a manifestation of a new form of $\mathrm{CH}$, involving the coordinated cycling of blood flow in dozens or hundreds of vessels; could there be individual vessels within regions of MRH that have cycling blood flow? An important aspect of MRH is its ability to account for hypoxia-related metastasis, and of course the lethality of most cancers is ultimately related to invasion and distant spread. Additionally, the multi-mm dimensions of MRH are perfectly adapted to the voxel size of PET imaging which is of the order of 64 $\mathrm{mm}^{3}$ for modern instruments. So the key question (for which we presently do not have an answer) is whether the pharmacological properties of imaging drugs are critical for adequate diffusion and binding in these large regions (i.e. whether the extended longitudinal vascular gradients will result in depletion of imaging agents along their path, just as with oxygen and other highly metabolized drugs). More generally, this raises the issue of blood flow as a related variable in the production of hypoxia. In fact, many papers outside the field of radiation biology discuss hypoxia as if it were generally related to overall poor blood flow (in contrast to the earliest definitions of DLH as related to blood vessel distribution, or microscopic local aberrations in flow caused by $\mathrm{CH}$ ). The current thinking related to blood flow from the perspective of magnetic resonance imaging is that DCE-MRI should be able to detect regions of hypoxia based on their inadequate blood flow. However, since the key parameter from this type of imaging ( $\mathrm{K}_{\text {trans }}$ ) has a dual dependence on both flow and permeability, it is possible to confuse high permeability, as is observed in MRH, with high blood flow. In a very preliminary investigation, we found no relationship between $\mathrm{K}_{\text {trans }}$ and the ability of Hoechst 33342 to label vasculature in tumors susceptible to MRH (20). Our only general information related to this topic is that EF5 is able to label such regions by IHC or PET $(20-22,25)$.

We suggest that the capability to independently measure blood flow is very important in dissecting 'types' of hypoxia. The gold standard for measuring blood flow is dynamic scanning of O-15-labelled water. In a pioneering study by Komar et al, it was shown that dynamic scanning of F-18-labelled EF5 provided images of similar quality to those of labeled water in patients with $\mathrm{H} \& \mathrm{~N}$ cancer (75). This, combined with other advantages of dynamic scanning suggest a dual use for imaging agents such as EF5 (30, 37). EF5's high lipophilicity ensures a rapid equilibration for all tissues, thus allowing this capability. It remains to be seen whether more hydrophilic agents, with much longer equilibration times between blood and tissue are equally capable in this regard. From a prognostic standpoint, 
there is a fascinating study by the Turku group showing that H\&N tumors with high blood flow are most susceptible to treatment failure (76).

The fact that none of the current methods can track $\mathrm{CH}$ is problematic since physical targeting strategies (e.g. image-guided radiation therapy) would be useless if hypoxia in tumors were moving from one spot to the next and/or between the times of imaging and therapy, somewhat akin to the 'whack-a-mole' game (20). Some investigators feel that $\mathrm{CH}$ is of overwhelming importance and fundamentally cannot be targeted through imaging techniques $(77,78)$. This would even affect molcecular assays since one would have to consider both the spatial \& temporal changes of hypoxia and the time between HIF induction or depletion (very rapid) and changes in synthesis of its downstream targets (several hrs). Much additional work is required in these areas.

\section{Optimizing Clinical Trials}

Since a finding of hypoxia is hypothesized to portend a poor prognosis, we considered high and/or extensive EF5 binding as a 'bad news' factor for patients. Indeed, in some very limited trials, this was found to be the case $(22,79,80)$. Similarly, in very carefully performed Eppendorf studies, using ultrasound guidance, Brizel was able to identify patients with hypoxic tumors that responded very poorly to standard therapy $(11,81)$.

To date, identification of patients who will do very poorly has seldom been accomplished by NII. The most dramatic positive example of such predictive capability has been for the compound FETNIM in esophageal cancer (82). In this study, 11 of 14 tumors with uptake responded poorly and only 1 of 14 tumors without uptake failed. A much more common finding has been an ambiguous determination of patients whose tumors will fail to respond. Several examples are summarized in a review paper by Horsman et al (46). In one of the most thorough prognostic investigations of NII yet published - using FAZA - Mortensen et al found 1 of 17 patients to recur in tumors that were negative for FAZA uptake, but only 7 failures in 25 patients that had positive uptake (83). It is critical to appreciate that this result, while very significant in terms of outcome, has little value in determining which patients should be receiving anti-hypoxia therapy. Lack of FAZA uptake appears to be a 'good news' assay but how this relates to hypoxia is unclear.

Despite the various knowledge gaps indicated in the previous sections, an indirect test of the overall principles has been made in a clinical trial designed to incorporate both PET imaging and bioreductive therapy. In the trans-Tasman trial of tirapazamine, a bioreductively activated hypoxia cytotoxin, a subgroup of patients was diagnosed by PET imaging with FMISO (84). Thus there were patients with FMISO positive or negative images, and portions of each subset either received or did not receive tirapazamine, in addition to standard therapy. On the assumption that FMISO indicated the hypoxia status of each tumor, one might have expected patients receiving tirapazaine with aerobic tumors to do the best, and patients not receiving tirapazamine but having hypoxic tumors to do the worst. Although the latter expectation was met, the patients that did the best were the ones that had hypoxic tumors and received tirapazamine. Although this apparent anomaly was not commented on in the initial paper our interpretation of the result was that the FMISO imaging was not only selecting for hypoxic tumors but also high nitroreductase levels - that 
is, a mildly hypoxic tumor with high nitroreductase might appear the same on PET imaging (and respond equally well or even better to tirapazamine) as a severely hypoxic tumor with low nitroreductase levels (30). This important trial was the first to indirectly suggest the importance of tumor nitroreductase levels and even more important allowed for the possibility (originally suggested by J. Martin Brown) that it might be possible to exploit tumor hypoxia with suitable therapy $(85,86)$. It also validated the concept of the need to target tumor cells at higher than severely hypoxic levels since tirapazamine has an unusually high $\mathrm{K}_{\mathrm{m}}$ for inhibition of toxicity by oxygen (87). Unfortunately, in the follow up study, FMISO-based PET imaging was not continued and some unexpected toxicities of tirapazamine occurred, thus eliminating this promising drug from further study. This was doubly unfortunate since the continued use of FMISO-PET might also have identified patients likely to experience toxicity (for example those that experienced abnormally high uptake of FMISO in normal tissues). Information of this sort would require PET imaging of the major organs (or at least a major muscle since muscle cramping was associated with tirapazamine toxicity - 88), and this is difficult with hydrophilic drugs such as FMISO due to high rates of excretion via kidney and bladder, and slow equilibration between blood and tissue.

Despite some problems, the trans-Tasman sub-group study has set the standard for what an optimal interventional trial should look like. Both imaging agent and the therapy agent appeared to have similar requirements in terms of oxygen and nitroreductase levels, and the cancer type studied ( $\mathrm{H} \& \mathrm{~N} \mathrm{Ca}$ ) was known to suffer from therapy resistance due to hypoxia. The Auckland University group has spent a great deal of effort in the analysis and problem solving for the next generation of drugs for such testing. As indicated above, they have been instrumental in elucidating mechanisms of nitroreductase activity. They have also been developing various therapeutic strategies that could prove superior to tirapazamine by developing agents with similar bioreductive characteristics, but improved tumor diffusional access and reduced toxicity - in keeping with the present discussions, their lead compound (SN30000) is more lipophilic than tirapazamine (51). They have also tested whether their bioreductive cytotoxins have the same enzyme requirements for reduction as the hypoxia imaging agent EF5. Despite the fact that both their next generation analog of tirapazamine and a prodrug with potential bystander effect have different chemistries than 2-

nitroimidazoles, the enzyme requirements for all three drugs appear to be nearly identical (55). The reasons for this are not yet clear but it is possible that they are all 'pure' bioreductives in the sense that there appears to be absolutely no non-oxygen dependent metabolism involved. This likely implies an absolute specificity for one-electron reductases (rather than 2-electron reductases that would be oxygen independent). Unfortunately, an unexpected toxicity for the prodrug PR-104 was traced to a 2-electron reduction by an enzymes uniquely activating this but not other bioreductives (52). Despite what appears to be a perfect marriage of imaging and therapeutic efficacy, these drug combinations have not proceeded to definitive trials, though EF5 has shown excellent diagnostic and predictive information for SN30000 in a rat-xenograft model of lung cancer (89).

A trial is ongoing at the University of Pennsylvanian that seeks to reverse hypoxia in H\&N tumors using the drug nelfinavir. This is a novel approach that may assist the process of re- 
oxygenation that can occur with fractionated therapy. Patients will be receiving PET scans of F18-labelled EF5 before and after the nelfinavir treatment, just prior to radiotherapy (90).

It has been suggested that dose-painting of hypoxia, using image-guided radiotherapy, might be an appropriate use for NII of hypoxia (91-94). Perhaps the optimal use of this method might occur in $\mathrm{H} \& \mathrm{~N}$ cancer where it has often been found that primary tumor versus nodes may have greatly contrasting levels of hypoxia (75). Similarly, the extraordinary sensitivity and specificity of the FETNIM result for esophageal cancer may provide an optimal test bed for anti-hypoxia therapies (82). Since it is known that the very hydrophilic nature of this agent might limit its access generally, this trial may have identified a cancer type that allows efficient access of hydrophilic drugs. Yet another promising approach would be to apply the kinetic analyses suggested by Thorwarth to tumors such as sarcomas, where hypoxia is strongly associated with metastases (11). It would be valuable to investigate whether the type of hypoxia associated with low perfusion is similar to what we have described as MRH. This would provide the first evidence that specific hypoxia sub-types could be imaged, and this might allow many new uses for NII.

\section{Abbreviations Used}

EF5

[2-(2-nitro-1-H-imidazol-1-yl)-N-(2,2,3,3,3-pentafluoropropyl)-acetamide]

FMISO 1-(2-nitro-1-H-imidazol-1-yl)-3-fluoro-2-propan-2-ol

FETA [2-(2-nitro-1-H-imidazol-1-yl)-N-(2-fluoroethyl)-acetamide

FETNIM 1-(2-nitro-1-H-imidazol-1-yl)-4-fluoro-butane-2,3-diol

FAZA 1-(5-fluoro-5-deoxy-a-D-arabinofuranosyl)-2-nitroimidazole

IAZGP 1-(6-deoxy-6-iodo-b-D-galactopyranosyl)-2-nitroimidazole

IHC immunohistochemistry

SA specific activity

NII Non-Invasive Imaging

PET Positron Emission Tomography

POR cytochrome P450 reductase

IGRT Image-guided Radiation Therapy

IMRT Intensity-Modulated Radiation Therapy

DLH diffusion-limited hypoxia

CH cycling hypoxia

MRH macroscopic regional hypoxia

\section{References}

1. Evans SM, Schrlau A, Chalian AA, et al. Oxygen levels in normal and previously irradiated human skin as assessed by EF5 binding. J Invest Dermatol. 2006; 126:2596-2606. [PubMed: 16810299] 
2. Tuttle SW, Maity A, Oprysko PR, et al. Detection of reactive oxygen species via endogenous oxidative pentose phosphate cycle activity in response to oxygen concentration: Implications for HIF-1 stability under moderate hypoxia. J Biol Chem. 2007; 282:36790-36796. [PubMed: 17666400]

3. Koch, CJ. "Competition Between Radiation Protectors and Radiation Sensitizers in Mammalian Cells". In: Nygaard, OF.; Simic, MG., editors. Radioprotectors and Anticarcinogens. NY: Academic press; 1983. p. 275-296.

4. Thomlinson RH, Gray LH. The histological structure of some human lung cancers and the possible implications for radiotherapy. Br J Cancer. 1955; 9:539-579. [PubMed: 13304213]

5. Varghese AJ, Whitmore GF. Binding to cellular macromolecules as a possible mechanism for the cytotoxicity of misonidazole. Cancer Res. 1980; 40:2165-2169. [PubMed: 6155991]

6. Wardman P. Molecular structure and biological activity of hypoxic cell radiosensitizers and hypoxic specific cytotoxins. Advanced Topics on Radiosensitizers of Hypoxic Cells. 1982:49-75.

7. Coleman CN, Wasserman TH, Urtasun RC, et al. Final report of the phase I trial of the hypoxic cell radiosensitizer SR 2508 (etanidazole) Radiation Therapy Oncology Group 83-03. Int J radiat Oncol Biol Phys. 1990; 18:389-393. [PubMed: 2154420]

8. Dische S. Chemical sensitizers for hypoxic cells: a decade of experience in clinical radiotherapy. Radioth Oncol. 1985; 3:97-115.

9. Hockel M, Knoop C, Schlenger $\mathrm{K}$, et al. Intratumor $\mathrm{pO}_{2}$ predicts survival in advanced cancer of the uterine cervix. Radiotherapy and Oncology. 1993; 26:45-50. [PubMed: 8438086]

10. Hockel M, Schlenger K, Aral B, et al. Association between tumor hypoxia and malignant progression in advanced cancer of the uterine cervix. Cancer Res. 1996; 56:4509-4515. [PubMed: 8813149]

11. Brizel DM, Scully SP, Harrelson JM, et al. Tissue oxygenation predicts for the likelihood of distant metastases in human soft tissue sarcoma. Cancer Res. 1996; 56:941-943. [PubMed: 8640781]

12. Fyles AW, Milosevic M, Wong R, et al. Oxygenation predicts radiation response and survival in patients with cervix cancer. Radioth and Oncol. 1998; 48:149-156.

13. Stone HB, Brown JM, Phillips TL, et al. Oxygen in human tumors: Correlations between methods of measurement and response to therapy. Radiat Res. 1993; 136:422-434. [PubMed: 8278585]

14. Nordsmark M, Bentzen SO, Rudat V, et al. Prognostic value of tumor oxygenation in 397 head and neck tumors after primary radiation therapy. An international multi-center study. Radioth and Oncol. 2005; 77:18-24.

15. Nordsmark M, Loncaster J, Aquino-Parsons C, et al. Measurements of hypoxia using pimonidazole and polarographic oxygen-sensitive electrodes in human cervix carcinomas. Radiother Oncol. 2003; 67:35-44. [PubMed: 12758238]

16. Brown JM. Evidence for acutely hypoxic cells in mouse tumours, and a possible mechanism of reoxygenation. Brit J Radiol. 1979; 52:650-656. [PubMed: 486895]

17. Chaplin DJ, Durand RE, Olive PL. Acute hypoxia in tumors: Implications for modifiers of radiation effects. Int J Radiat Biol Phys. 1986; 12:1279-1282.

18. Chaplin DJ, Olive PL, Durand RE. Intermittent blood flow in a murine tumor: radiobiological effects. Cancer Res. 1987; 47:597. [PubMed: 3791244]

19. Trotter MJ, Olive PL, et al. Effect of vascular marker Hoechst 33342 on tumour perfusion and cardiovascular function in the mouse. Brit J Cancer. 1990; 62:903-908. [PubMed: 2257217]

20. Koch CJ, Jenkins WT, Jenkins KW, et al. Mechanisms of blood flow and hypoxia production in rat 9L-epigastric tumors. Tumor Microenv \& Ther. 2012; 1:1-13.

21. Evans SM, Hahn S, Pook DR, et al. Hypoxia in human intraperitoneal and extremity sarcomas. Int J Radiat Oncol Biol Phys. 2000; 49:587-596. [PubMed: 11173159]

22. Evans SM, Jenkins KW, Chen HI, et al. The relationship between hypoxia, proliferation and outcome in patients with de novo glioblastoma: a pilot study. Translat Oncol. 2010; 3:160-169.

23. Dewhirst MW, Ong ET, Braun RD, et al. Quantification of longitudinal tissue $\mathrm{pO}_{2}$ gradients in window chamber tumours: impact on tumour hypoxia. Brit J Cancer. 1999; 79:1717-1722. [PubMed: 10206282] 
24. Evans SM, Koch CJ, Joiner B, et al. Identification of hypoxia in cells and tissues of epigastric 9L rat glioma using EF5 [2-(2-nitro-1H-imidazol-1-yl)-N-(2,2,3,3,3-pentafluoropropyl)acetamide]. Brit J Cancer. 1995; 72:875-882. [PubMed: 7547234]

25. Chitneni SK, Bida GT, Zalutsky MR, et al. Comparison of the hypoxia PET tracer ${ }^{18} \mathrm{~F}-\mathrm{EF} 5$ to immunohistochemical marker EF5 in 3 different human tumor xenograft models. J Nuc Med. 2014; 55:1192-1197.

26. Koch CJ. Measurement of Absolute Oxygen Levels in Cells and Tissues using Oxygen Sensors and the 2-nitroimidazole EF5. Meth Enzymol - Antioxidants and Redox Cycling. 2002; 353:3-31.

27. Aebersold DM, Burri P, Beer KT, et al. Expression of hypoxia-inducible factor-1a: a novel predictive and prognostic parameter in the radiotherapy of oropharyngeal cancer. Cancer Res. 2001; 61:2911-2916. [PubMed: 11306467]

28. Vahidi N, Clarkson RB, Liu KJ, et al. In vivo and in vitro EPR oximetry with fusinite: a new coalderived, particulate EPR probe. Mag Res in Med. 1994; 31:139-146.

29. Yasui H, Matsumoto S, Devasahayam N, et al. Low field magnetic resonance imaging to visualize chronic and cycling hypoxia in tumor-bearing mice. Cancer Res. 2010; 15:6427-6436. [PubMed: 20647318]

30. Koch, CJ.; Evans, SM. Non-Invasive Imaging of Hypoxia - Challenges and Opportunities. In: Chen, X., editor. Molecular Imaging Probes for Cancer Research. NJ: World Scientific; 2010. p. 285-313.Chapter 10

31. Overgaard J, Hansen HS, Overgaard M, et al. A randomized double-blind phase III study of nimorazole as a hypoxic radiosensitizer of primary radiotherapy in supraglottic larynx and pharynx carcinoma. Results of the Danish Head and Neck Cancer Study (DAHANCA) Protocol 5-85. Radioth \& Oncol. 1998; 46:135-146.

32. Mohindra JE, Rauth AM. Increased killing by metronidazole and nitrofurazone of hypoxic compared to aerobic mammalian cells. Cancer Res. 1976; 36:930-936. [PubMed: 1253180]

33. Sridhar R, Koch CJ, Sutherland RM. Cytotoxicity of two nitroimidazole radiosensitizers in an in vitro tumor model. Int J Radiat Oncol Biol Phys. 1976; 1:1149-1157. [PubMed: 993091]

34. Varghese AJ, Gulyas S, Mohindra JK. Hypoxia-dependent reduction of 1-(2-nitro-1-imidazolyl)-3 -methoxy-2-propanol by Chinese hamster ovary cells and KHT tumor cells in vitro and in vivo . Cancer Res. 1976; 36:3761-3765. [PubMed: 986241]

35. Chapman JD. Hypoxic sensitizers - implications for radiation therapy. New Eng J of Med. 1979; 301:1429-1432. [PubMed: 229413]

36. Iyer RV, Haynes PT, Schneider RF, et al. Marking hypoxia in rat prostate carcinomas with b-D[125I] azomycin galactopyranoside and [99mTc]HL-91: Correlation with microelectrode measurements. J Nucl Med. 2001; 42:337-344. [PubMed: 11216534]

37. Krohn KA, Link JM, Mason RP. Molecular imaging of hypoxia. J Nucl Med. 2008; 49:129S148S. [PubMed: 18523070]

38. Miller GG, Best MW, Franko AJ, et al. Quantitation of hypoxia in multicellular spheroids by video image analysis. Int J Radiat Oncol Biol Phys. 1989; 16:949-952. [PubMed: 2649466]

39. Hodgkiss RJ, Jones G, Long A, et al. Flow cytometric evaluation of hypoxic cells in solid experimental tumours using fluorescence immunodetection. Br J Cancer. 1991; 63:119-125. [PubMed: 1989649]

40. Lord EM, Harwell LW, Koch CJ. Detection of hypoxic cells by monoclonal antibody recognizing 2-nitroimidazole adducts. Cancer Res. 1993; 53:5271-5276.

41. Varia MA, Calkins-Adams DP, Rinker LH, et al. Pimonidazole: a novel hypoxia marker for complementary study of tumor hypoxia and cell proliferation in cervical carcinoma. Gynecologic Oncol. 1998; 71:270-277.

42. Dolbier WR, Li A-R, Koch CJ, et al. [18F]-EF5, a marker for PET detection of hypoxia: Synthesis of precursor and a new fluorination procedure. Appl Rad and Isot. 2001; 54:73-80.

43. Josse O, Labar D, Georges B, et al. Synthesis of [F-18]-labeled EF3 [2-(2-nitroimidazol-1-yl)-N(3,3,3-trifluoropropyl)-acetamide], a marker for PET detection of hypoxia. Bioorg Med Chem. 2001; 9:665-675. [PubMed: 11310602]

44. Lee ST, Scott AM. Hypoxia positron emission tomography imaging with ${ }^{18}$ F-Fluoromisonidazole. Sem Nuc Med. 2007; 37:451-461. 
45. Ballinger JR. Imaging hypoxia in tumors. Seminars Nucl Med. 2001; 31:321-329.

46. Horsman MR, Mortensen LS, Petersen JB, et al. Imaging hypoxia to improve radiotherapy outcome. Nature Rev Clin Oncol. 2012; 9:674-687. [PubMed: 23149893]

47. Koch, CJ. "The reductive activation of nitroimidazoles; modification by oxygen and other redoxactive molecules in cellular systems". In: NATO Series, A.; Adams, GE.; Breccia, A.; Fielden, EM.; Wardman, P., editors. Selective Activation of Drugs by Redox Processes. Vol. 198. NY: Plenum Press; 1990. p. 237-247.

48. Chapman, JD.; Lee, J.; Meeker, BE. Adduct formation by 2-nitroimidazole drugs in mammalian cells: optimization of markers for tissue oxygenation. In: NATO Series, A.; Adams, GE.; Breccia, A.; Fielden, EM.; Wardman, P., editors. Selective Activation of Drugs by Redox Processes. Vol. 198. NY: Plenum Press; 1990. p. 313-323.

49. Koch CJ, Evans SM, Lord EM. Oxygen dependence of cellular uptake of EF5 [2-(2-nitro-1Himidazol-1-yl)-N-(2,2,3,3,3-pentafluoroproply)acetamide] : analysis of drug adducts by fluorescent antibodies vs bound radioactivity. Br J Cancer. 1995; 72:869-874. [PubMed: 7547233]

50. Koch CJ. Importance of Antibody Concentration in the Assessment of Cellular Hypoxia by Flow Cytometry: EF5 and Pimonidazole. Radiat Res. 2008; 169:677-688. [PubMed: 18494550]

51. Wilson WR, Hay MP. Targeting hypoxia in cancer therapy. Nature Rev Cancer. 2011; 11:393-410. [PubMed: 21606941]

52. Guise CP, Abbattista MR, Singleton RS, et al. The bioreductive prodrug PR-104A is activated under aerobic conditions by human aldo-keto reductase 1C3. Cancer Res. 2010; 70:1573-1584. [PubMed: 20145130]

53. Busk M, Horsman MR, Jakobsen S, et al. Cellular uptake of PET tracers of glucose metabolism and hypoxia and their linkage. Eur J Nuc Med Mol Imaging. 2008; 35:2294-2203.

54. Koch CJ. A thin-film culturing technique allowing rapid gas-liquid equilibration (6 seconds) with no toxicity to mammalian cells. Radiat Res. 1984; 97:434-442. [PubMed: 6695057]

55. Guise CP, Abbattista MR, Tipparaju SR, et al. Diflavin oxidoreductases activate the bioreductive prodrug PR-104A under hypoxia. Mol Pharmacol. 2012; 81:31-40. [PubMed: 21984255]

56. Prosser GA, Copp JN, Syddall SP, et al. Discovery and evaluation of Escherichia coli nitroreductases that activate the anti-cancer prodrug CB1954. Biochem Pharmacol. 2010; 79:678687. [PubMed: 19852945]

57. Raleigh JA, Koch CJ. The importance of thiols in the reductive binding of 2-nitroimidazoles to macromolecules. Biochem Pharmacol. 1990; 40:2457-2464. [PubMed: 2176499]

58. Koch CJ, Raleigh JA. Radiolytic reduction of protein and non-protein disulfides in the presence of formate : a chain reaction. Arch Biochem Biophys. 1991; 287:75-84. [PubMed: 1897997]

59. McClelland RA, Panicucci R, Rauth AM. Products of the reductions of 2-nitroimidazoles. J Am Chem Soc. 1987; 109:4308-4314.

60. Koch CJ, Stobbe CC, Baer KA. Metabolism induced binding of 14C-misonidazole to hypoxic cells: kinetic dependence on oxygen concentration and misonidazole concentration. Int J Radiat Oncol Biol Phys. 1984; 10:1327-1332. [PubMed: 6469755]

61. Laughlin KM, Evans SM, Jenkins WT, et al. Biodistribution of the nitroimidazole EF5 [2-(2nitro-1H-imidazole-1yl)-N-(2,2,3,3,3-pentafluoropropyl)-acetamide] in mice bearing subcutaneous EMT6 tumors. J Pharmacol Exptl Therapeut. 1996; 277:1049-1057.

62. Evans SM, Koch CJ. Prognostic significance of tumor oxygenation in humans. Cancer Letters. 2003; 195:1-16. [PubMed: 12767506]

63. Wang J, Foehrenbacher A, Su J, et al. The 2-nitroimidazole EF5 is a biomarker for oxidoreductases that activate the bioreductive prodrug CEN-209 under hypoxia. Clin Cancer Res. 2012; 18:16841695. [PubMed: 22167409]

64. Rasey JS, Koh W-J, Evans ML, et al. Quantifying regional hypoxia in human tumors with positron emission tomography of [18F]fluoromisonidazole: a pretherapy study of 37 patients. Int J Radiat Oncol Biol Phys. 1996; 36:417-428. [PubMed: 8892467]

65 . Thorwarth D, Eschmann S-M, Scheiderbaur J, et al. Kinetic analysis of dynamic 18Ffluoromisonidazole PET correlates with radiation treatment outcome in head-and-neck cancer. BMC Cancer. 2005; 5:152. [PubMed: 16321146] 
66. O’Donoghue JA, Guillem JG, Schoder H, et al. Pilot study of PET imaging of 124I-iodoazomycin galactopyranoside (IAZGP), a putative hypoxia imaging agent, in patients with colorectal cancer and head and neck cancer. EJNMMI Res. 2013; 3:42. [PubMed: 23731770]

67. Workman P. Pharmacokinetics of hypoxic cell radiosensitizers. Cancer Clin Trials. 1980; 3:237251. [PubMed: 7438321]

68. Mees G, Maes A, Sathekge M, et al. Radiolabelled probes targeting tumor hypoxia for personalized medicine. Curr Pharmaceut Dev. 2014; 20:2308-23018.

69. Cascieri JJ, Graham MM, Rasey JS. A modelling approach for quantifying tumor hypoxia with [F-18]fluoromisonidazole PET time-activity data. Med Phys. 1995; 22:1127-1139. [PubMed: 7565388]

70. Carlin S, Humm JL. PET of hypoxia: current and future perspectives. J Nuc Med. 2012; 53:11711174.

71. Hicks KO, Fleming Y, Siim BG, et al. Extravascular diffusion of tirapazamine: effect of metabolic consumption assessed using the multicellular layer model. Int J Rad Onc Biol Phys. 1998; 42:641649.

72. Sutherland RM. Cell and environmental interactions in tumor microregions: the multicellular spheroid model. Science. 1988; 240:177-184. [PubMed: 2451290]

73. Gronroos T, Eskola O, Lehtio K, et al. Pharmacokinetics of [18F]FETNIM: a potential marker for PET. J Nucl Med. 2001; 42:1397-1404. [PubMed: 11535732]

74. Koch CJ, Hahn SM, Rockwell KJ, et al. Pharmacokinetics of the 2-nitroimidazole EF5 [2-(2nitro-1-H-imidazol-1-yl)-N-(2,2,3,3,3-pentafluoropropyl)acetamide] in human patients: implications for hypoxia measurements in vivo. Cancer Chemoth Pharmacol. 2001; 48:177-187.

75. Komar G, Seppänen M, Eskola O, et al. 18F-EF5: A new PET tracer for imaging hypoxia in head and neck cancer. J Nuc Med. 2008; 49:1-8.

76. Lehtio K, Eskola O, Viljanen T, et al. Imaging perfusion and hypoxia with PET to predict radiotherapy response in head-and-neck cancer. Int J Rad Oncol Biol Phys. 2004; 35:975-980.

77. Cardenas-Navia LI, Mace D, Richardson RA, et al. The Pervasive Presence of Fluctuating Oxygenation in Tumors. Cancer Res. 2008; 68:5812-5819. [PubMed: 18632635]

78. Rofstad EK, Gaustad JV, Egeland TAM, et al. Tumors exposed to acute cycling hypoxic stress show enhanced angiogenesis, perfusion and metastatic dissemination. Int J Cancer. 2010; 127:1535-1546. [PubMed: 20091868]

79. Evans SM, Hahn S, Pook DR, et al. Detection of hypoxia in human squamous cell carcinoma by EF5 binding. Cancer Res. 2000; 60:2018-2024. [PubMed: 10766193]

80. Evans SM, Fraker DL, Hahn SM, et al. EF5 binding and clinical outcome in human soft tissue sarcomas. Int J Radiat Oncol Biol Phys. 2006; 64:922-927. [PubMed: 16458778]

81. Brizel DM, Sibley GS, Prosnitz LR, et al. Tumor hypoxia adversely affects the prognosis of carcinoma of the head and neck. Int J Radiat Oncol Biol Phys. 1997; 38:285-289. [PubMed: 9226314]

82. Yue J, Yang Y, Cabrera AR, et al. Measuring tumor hypoxia with 18F-FETNIM in esophageal squamous cell carcinoma: a pilot clinical study. Dis Esophagus. 2012; 25:54-61. [PubMed: 21595781]

83. Mortensen LS, Johansen J, Kallehauge J, et al. FAZA PET/CT hypoxia imaging in patients with squamous cell carcinoma of the head and neck treated with radiotherapy: Results from the Dahanca 24 trial. Radioth and Oncol. 2012; 105:14-20.

84. Rischin D, Hicks RJ, Fisher R, et al. Prognostic significance of [18F]-misonidazole positron emission tomography-detected tumor hypoxia in patients with advanced head and neck cancer randomly assigned to chemoradiation with or without tirapazamine: a substudy of trans-Tasman Radiation Oncology Group Study 98.02. J Clin Oncol. 2006; 24:2098-2104. [PubMed: 16648512]

85. Brown JM, Giaccia AJ. The unique physiology of solid tumors: Opportunities (and problems) for cancer therapy. Cancer Res. 1998; 58:1408-1416. [PubMed: 9537241]

86. Brown JM. Imaging tumor sensitivity to a bioreductive prodrug: two for the price of one! Clin Cancer Res. 2012; 18:1487-1489. [PubMed: 22317761] 
87. Koch CJ. The unusual dependence on oxygen concentration of toxicity by SR-4233 [3amino-1,2,4-benzotriazine-1,4-dioxide]: an hypoxic cell toxin. Cancer Res. 1993; 53:3992-3997. [PubMed: 8358728]

88. Rischin D, Peters L, Hicks R, et al. Phase I trial of concuurent tirapazamine, cisplatin, and radiotherapy in patients with advanced head and neck cancer. J Clin Oncol. 2001; 19:535-542. [PubMed: 11208848]

89. Chitneni SK, Bida GT, Yuan H, et al. 18F-EF5 PET imaging as an early response biomarker for the hypoxia-activated prodrug SN30000 combined with radiation treatment ina non-small cell lung cancer xenograft model. J Nuc Med. 2013; 54:1339-1346.

90. Gupta AG, Lee JH, Wilke WW, et al. Radiation response in two HPV-infected head-and-neck cancer cell lines in comparison to a non-infected cell line and relationship to signalling through AKT. Int J Radiat Oncol Biol Phys. 2009; 74:928-933. [PubMed: 19480971]

91. Ling CC, Humm J, Larson S, et al. Towards multidimensional radiotherapy (MD-CRT): biological imaging and biological conformality. Int J Radiat Oncol Biol Phys. 2000; 47:551-560. [PubMed: 10837935]

92. Grosu A-L, Souvatzoglou M, Roper B, et al. Hypoxia imaging with FAZA-PET and theoretical considerations with regard to dose painting for individualization of radiotherapy in patients with head and neck cancer. Int J Radiat Oncol Biol Phys. 2007; 69:541-551. [PubMed: 17869667]

93. Chao KS, Bosch WR, Mutic S, et al. A novel approach to overcome hypoxic tumor resistance: $\mathrm{Cu}$ ATSM-guided intensity-modulated radiation therapy. Int J Radiat Oncol Biol Phys. 2001; 49:1171-1182. [PubMed: 11240261]

94. Flynn RT, Bowen SR, Bentzen SM, et al. Intensity-modulated x-ray (IMXT) versus proton (IMPT) therapy for theragnostic hypoxia-based dose painting. Phys in Med and Biol. 2008; 53:4153-4167. [PubMed: 18635895] 


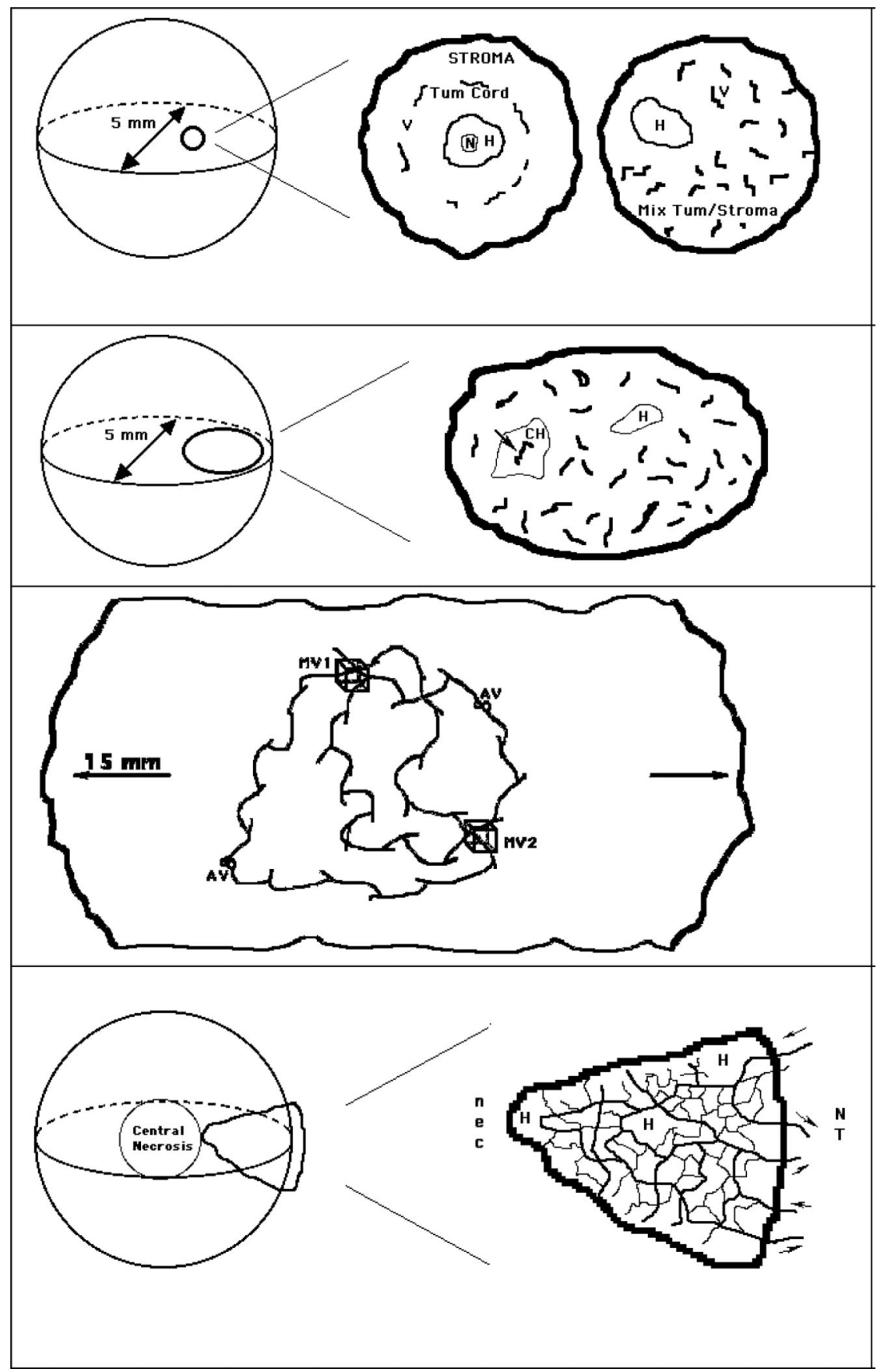

Figure 1.

a - Diffusion-Limited Hypoxia - This type of hypoxia $(\mathrm{H})$ occurs when the spatial heterogeneity of vessels causes small zones of tumor tissue to be too far from vessels for diffusion of oxygen. It was initially described in epithelial tumors where cords of tumor tissue are surrounded by vessels within the normal stroma (center) but is also found in nonepithelial tumors (right). Sometimes, necrosis $(\mathrm{N})$ is found in the center of a hypoxic zone. The centre and right panels are an expanded view of a $0.5 \mathrm{~mm}$ sphere from a $5 \mathrm{~mm}$ diameter tumor (left). 
b - Cycling Hypoxia - This type of hypoxia $(\mathrm{CH})$ occurs when blood flow in an isolated blood vessel cycles in an on-off pattern (arrow). It was proposed that two fluorescent DNAbinding dyes, when injected at different times, could label such vessels by demonstrating only one of the two dyes. A region of diffusion-limited hypoxia $(\mathrm{H})$ is also shown. Right panel represents an expanded view of a $1.5 \mathrm{~mm}$ ovoid from a $5 \mathrm{~mm}$ diameter tumor, left). c - In larger and particularly orthotopic tumors, blood travels over extended distances along unpaired vessels, which originate from co-opted normal-tissue vessels. This allows for a very heterogeneous mixture of vessels that can be carrying hypoxic or aerobic blood depending on the distance from the nearest arterial source. In this highly simplified diagram, two artery-vein pairs are depicted (AV) in a $15 \mathrm{~mm}$ diameter tumor. These produce various types of paths that may travel through volumes of interest (MV1 \& MV2). In this example MV1 is perfused by predominantly arterial blood, whereas MV2 is perfused by predominantly venous blood. Note the $15 \mathrm{~mm}$ scale, vs $5 \mathrm{~mm}$ for the other figures. $\mathrm{d}$ - In smaller $(5 \mathrm{~mm})$ murine tumors (as described in panels a \& b) vessels that arise from the normal tissue (NT) in the tumor periphery may be incapable of carrying oxygen and nutrients to the tumor interior. This can lead to macroscopic central necrosis (nec) - a feature often observed in such tumors. In this highly simplified diagram (expanded in right panel), a network of vessels is shown to originate from 2 arterioles and venules (arrows) outside a wedge-shaped portion of tumor. In an actual tissue section of such a region, many more 'vessels' would be observed since the network is not planar and the tissue section would cut randomly through these and other vessels/vessel-networks. Small regions of hypoxia $(\mathrm{H})$ are also shown. 


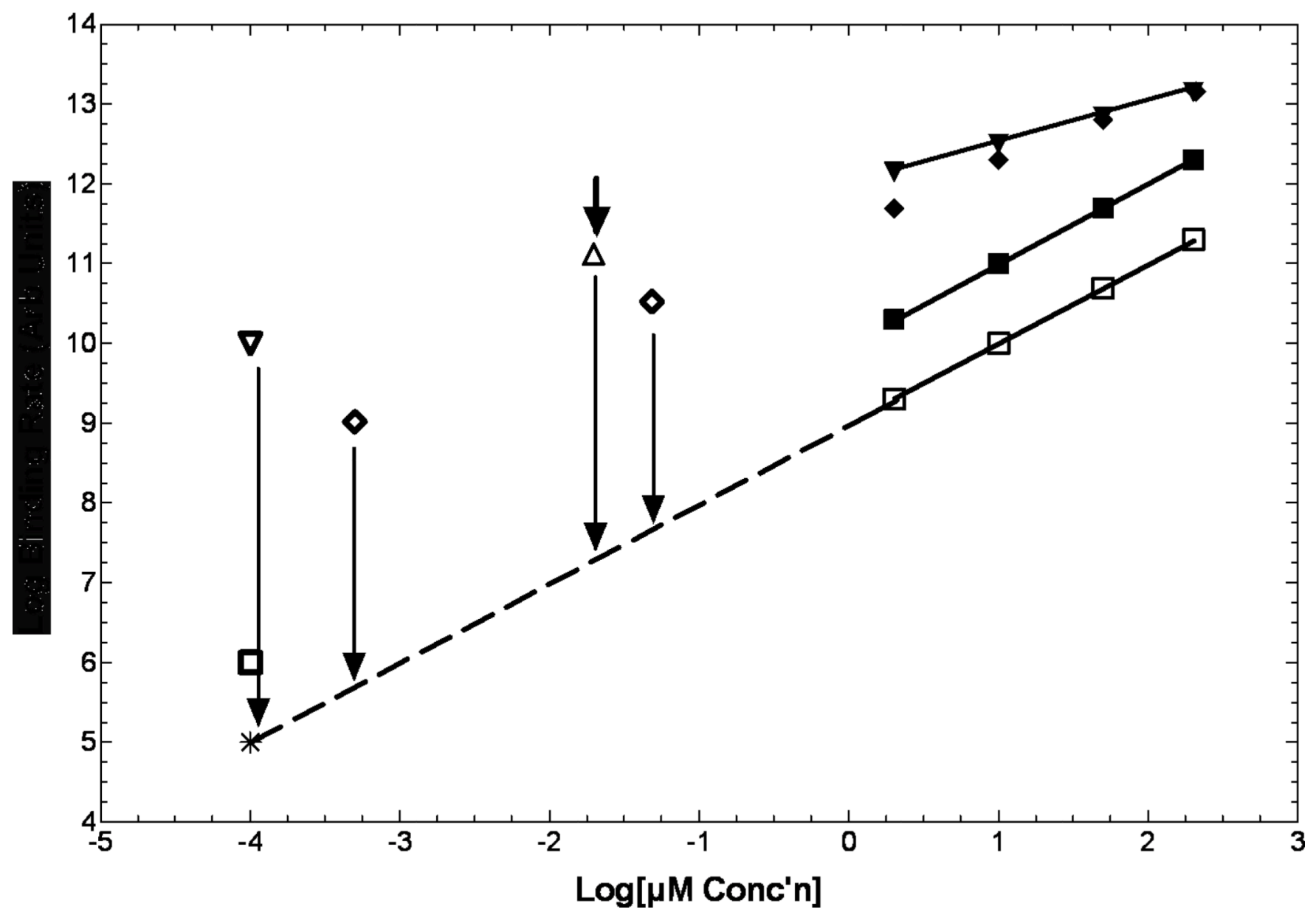

Figure 2.

Kinetics of binding are only known for a few compounds, and at $\mu \mathrm{M}$ to near $\mathrm{mM}$ concentrations (upper right). Representation of the shapes of these curves are illustrated for misonidazole (inverted closed triangles), EF5 (closed diamonds) and etanidazole (closed squares). All drugs tested revert to $1^{\text {st }}$ order dependence on drug concentration at low oxygen levels (open squares; $0.2 \%$, etanidazole). Shown at many logs lower concentrations are the low and moderate SA ${ }^{18} \mathrm{~F}-\mathrm{EF} 5$ (open diamonds), high SA ${ }^{18} \mathrm{~F}-\mathrm{FMISO}$ (open inverted diamond) and ${ }^{18} \mathrm{~F}$-fluoroetanidazole (open square). The only in vitro data in the high SA range is for ${ }^{18}$ F-FAZA (open triangle). No current information exists as to whether these extrapolated points mean anything, or what happens between anoxic binding and (for example) $0.2 \%$ oxygen (vertcal arrows). Note that this graph spans many decades of concentration for both axes. 


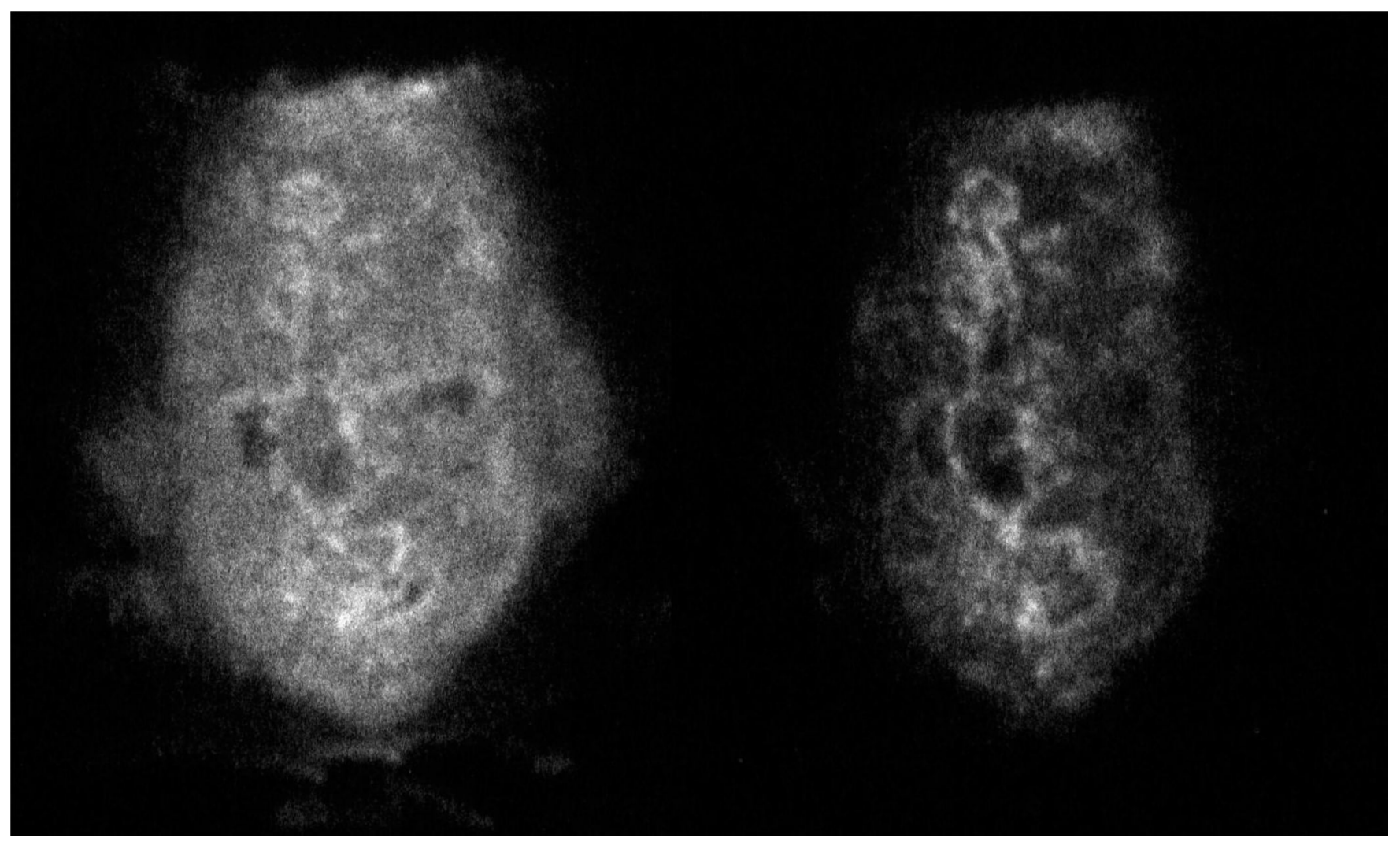

Figure 3.

Autoradiographs of R3230 mammary tumor from rat exposed to unlabelled plus labelled EF5, 3 hrs following drug injection. The pixel size of the autoradiograph imager (Fuji 3000) is 30 micrometers, so we used 30 micrometer thick adjacent sections (compared with 10 micrometer sections for IHC - see figure 4). On the left is shown a section that was simply dessicated in the cryostat before exposure to the phosphor plate, while on the right is shown an adjacent section that was fixed in ethanol before exposure. The panel on the left has similar contrast to that shown for a rat-xenograft in reference (25). Brightness for the right panel was enhanced by a factor of 4 to provide similar intensity levels. Size of tumor section $\sim 8 \mathrm{~mm} \times 15 \mathrm{~mm}$. 

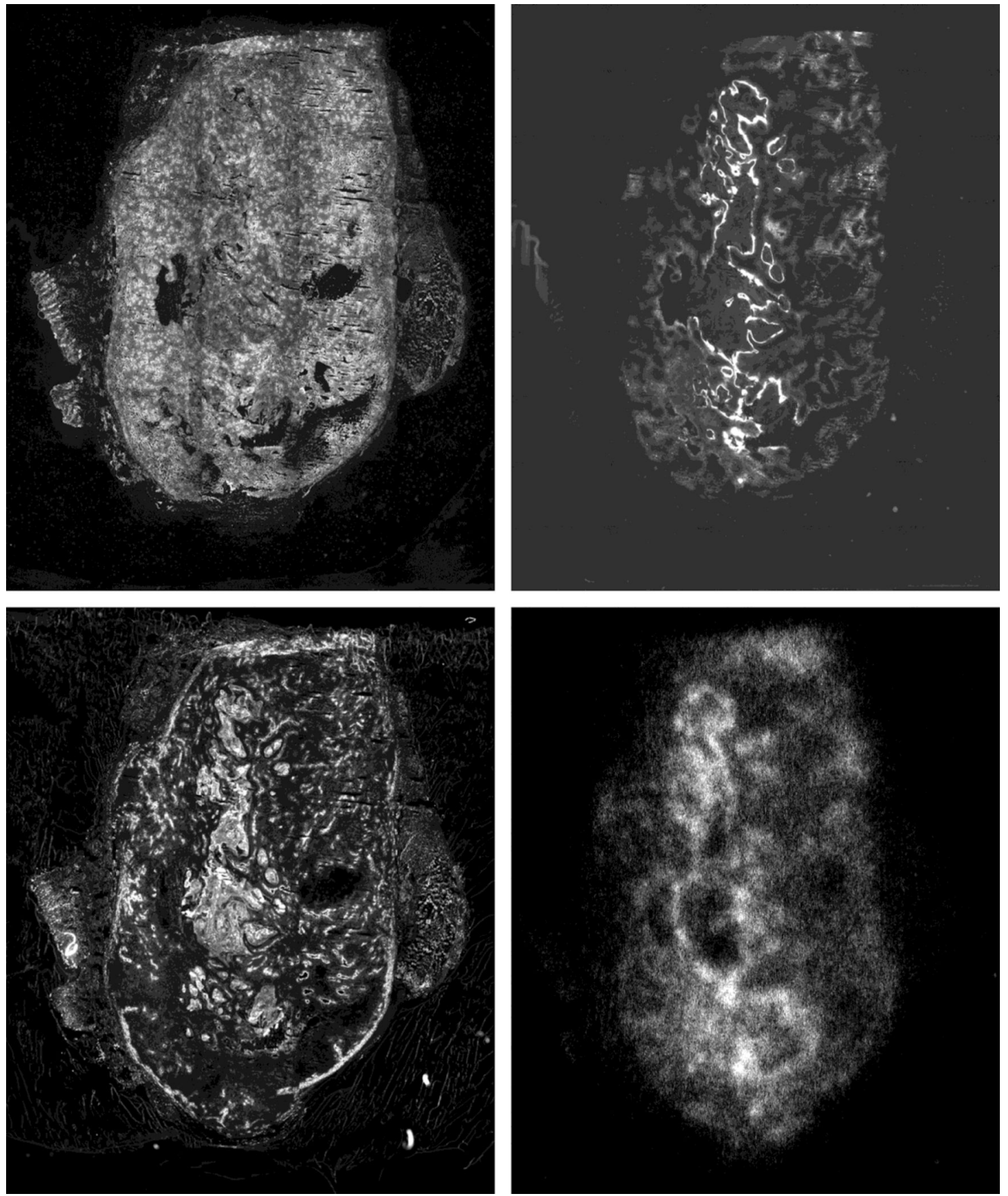

Figure 4.

3 additional $10 \mu \mathrm{m}$ thick (adjacent) sections were collected from the same tumor. Upper left shows 'flooded Hoechst' where all tissue is stained with Hoechst 33342 to indicate position of nuclei. This shows areas of necrosis or missing tissue. On the upper right is shown the IHC image for EF5 binding, which is very comparable to that for the rinsed AR section (lower right panel, copied from Fig 3). On the lower left is shown the image resulting from the in vivo injection of Hoechst 33342 dye. The positions of the main vessels are easily seen. Note the central region of uniformly high binding. This is normal tissue stroma which one 
typically sees in orthotopic tumors - the vascular system of normal tissue is such that essentially every normal cell in the body is labelled by injected Hoechst 33342 (Koch and Evans, unpublished data). 
Table 1

Semi-quantitative descriptors of 'hypoxia'.

\begin{tabular}{|l|l|l|l|}
\hline pO2 (mm of Hg) & Description & $\begin{array}{l}\text { \% Max. EF5 } \\
\text { Metab }\end{array}$ & Abs Bind Rate (aM/cell/hr) \\
\hline 76 & Physiological & 1 & 1.5 \\
\hline 19 & Mild Hypoxia & 3 & 5 \\
\hline 3.8 & Moderate Hypoxia & 10 & 15 \\
\hline 0.76 & Severe Hypoxia & 30 & 50 \\
\hline 0.0 & Anoxia & 100 & 150 \\
\hline
\end{tabular}

In order to calculate the absolute binding rate (column 4) cells were incubated at various oxygen levels in the presence of $100 \mu \mathrm{M} \mathrm{C14-labelled}$ EF5 and after various times, the cells were rinsed thoroughly, and treated with 5\% TCA to allow complete disruption with precipitation of macromolecules (26). The total binding rate (shown) consists of about $25 \%$ acid insoluble and $75 \%$ acid soluble metabolites. The rates shown are for the V79 Chinese hamster cells, which are similar to the average of several other cell lines (49). Since these cells have a mass of about $10^{\wedge}-12$ $\mathrm{kg}$, the rate under anoxia implies that the amount of metabolized drug is about 1.5 times the extracellular drug concentration, per hr. 


\section{Table 2}

Known 1-electron Nitroreductases and substrate $\mathrm{K}_{\mathrm{m}}$ 's

\begin{tabular}{|l|l|l|l|}
\hline Name & Origin & $\mathbf{K}_{\mathbf{m}}$ for CB1954* $(\mathbf{m M})$ & Reference \\
\hline NfsA & E Coli & 0.22 & $(55)$ \\
\hline NfsB & E Coli & 3.6 & $(55)$ \\
\hline NemA & E Coli & 0.06 & $(55)$ \\
\hline POR & mammalian & ND & $(56,63)$ \\
\hline MTRR & mammalian & ND & $(56)$ \\
\hline NDOR1 & mammalian & ND & $(56)$ \\
\hline NOS2A & mammalian & ND & $(56)$ \\
\hline
\end{tabular}

* Similar values have been found using EF5 as substrate (A.V. Patterson, personal communication) 